\section{A SHORT HISTORY OF THE EPIDEMIC INFECTIOUS DISEASES}

\author{
By E. W. Goodall, O.B.E., M.D. \\ John Bale, Sons \& Danielsson, Ltd. \\ Price 3/6.
}

In this small volume is told in a most interesting manner the story of the infectious fevers and of the most notable epidemics which have visited mankind.

By way of introduction the author traces the growth of the idea of infection from the time of Hippocrates to the present day. $\mathrm{He}$ shows how our modern ideas have gradually evolved from the most nebulous conceptions of the ancients by a process of ebb and flow, with an alternation of the belief in spontaneous generation and some specific infective factor (contagium vivum), until finally, in consequence of instrumental aids, the matter ceased to be one of the imaginings of the poet and became a palpable truth visible to the eye.

In his concluding chapter, Dr. Goodall discusses what lessons have been drawn throughout the ages from the different conceptions of infection in so far as they are evidenced by attempts at prevention. This also is a long story. Isolation of the sick man to prevent the spread of the disease has been practised for centuries. Quarantine was instituted during the 14th Century and in the 16th Century was so rigidly enforced that the penalty of death was imposed on those who deliberately left an infected area to enter an uninfected one. Isolation Hospitals had been long in existence for leprosy and plague before they were first employed in the case of other infections during the 18th Century. The discovery of the carrier of disease has revealed to us why isolation was limited in its value and to-day our most efficacious defence is the immunisation of the population. But even this is an old story. Inoculation against small pox is traditionally believed to date from pre-Christian times. Jenner, however, in 1796, inaugurated a new era for vaccination, and in 1881 and 1885 Pasteur showed the unlimited possibilities in this field when he introduced vaccination for the prevention of splenic fever and hydrophobia. Now-a-days enteric fever, plague, cholera, diphtheria and scarlet fever are similarly prevented.
As Dr. Goodall remarks, we should be proud of and grateful for all that has been done by our predecessors in the prevention of the visitations of these dire diseases. Nevertheless, he reminds us, as did Sophocles 2,400 years ago, of the "irrational wilfulness of human thought and action and a culpable negligence" to take advantage of the lessons we have learnt.

The volume is one which we most cordially commend to the notice of our readers.

\section{AIDS TO NEUROLOGY.}

\section{E. A. Blake Pritchard.}

Bailliere, Tindall \& Cox, London, 1934. Price 5/-.

For some years the need of an adequate textbook on diseases of the nervous system has been widely appreciated. Neurologists themselves have not been insensitive to this fact, and certain of them have lately been stimulated to rise to the occasion. The result has been the appearance within the past year of several monographs on nervous disorders and neuropathology. One of the latest additions to the invaluable "Students' Aids" series, is Dr. E. A. Blake Pritchard's "Aids to Neurology." This book is divided into five sections, dealing respectively with Symptomatology, the Peripheral Nervous System, the Spinal Cord, the Brain and the Autonomic Nervous System. The first of these, we feel, will be a difficult nut for most students to crack, for the author has failed to express himself in clear and simple terms, likely to be generally understood. The other four sections are much more simply written. A considerable amount of up-to-date information is imparted along conventional textbook lines. Herein, indeed, lies the weakness of this volume. It is much too big; 365 pages of fairly close print, containing the orthodox expositions in symptomatology, differential diagnosis, prognosis and treatment, scarcely conform to the requirements of a medical student wishing to get a useful working-knowledge of neurology. We feel this despite the author's claim that "completeness of detail has been made subordinate to the presentation of significant facts in reasonable perspective." On the other hand it is a good five shillings' worth. 